\title{
Análisis comparativo de la legislación en materia de las titulaciones de Técnico Deportivo y Técnico Deportivo Superior en la especialidad de deportes de invierno en España Comparative analysis of the legal system in the field of Sports Instructor certifications in winter sports in Spain \\ Joana Sans Osanz, Eduard Inglés Yuba \\ Universidad de Barcelona (España)
}

\begin{abstract}
Resumen. Estudios previos describen un auge en las actividades físicas en el medio natural y la consecuente necesidad de formar personal cualificado para dirigirlas. Aun así, el sistema educativo de profesionales de estas actividades aún hoy está siendo ordenado y estructurado. El presente artículo pretende comparar las normativas vigentes en las formaciones técnicas (técnico deportivo y técnico deportivo superior) de los deportes de invierno (esquí alpino, esquí de fondo y snowboard) y analizar las diferencias en la regulación en la España de las comunidades autónomas. Se ha analizado el contenido de la normativa estatal y el desarrollo normativo vigente de cada comunidad en materia de las titulaciones técnicas de la especialidad de deportes de invierno. En el año 2018, ocho comunidades han desarrollado normativa específica relativa a las titulaciones técnicas en la especialidad de los deportes de invierno, 18 años después de la publicación de la normativa estatal. Por lo general, se observa heterogeneidad en la regulación entre las diversas comunidades y también en comparación con la normativa estatal en aspectos tan relevantes como las capacidades profesionales, el ejercicio de la actividad profesional, las asignaturas cursadas, la distribución horaria, la carga lectiva total y la proporción de horas teóricas y prácticas. Es necesaria una homogeneización entre comunidades autónomas de la regulación de las titulaciones técnicas en deportes de invierno, con el objetivo de asegurar una línea convergente para el ejercicio profesional de estas modalidades en todo el territorio estatal.

Palabras clave. Técnico deportivo; Técnico deportivo superior; Actividades físicas en el medio natural; Deportes de invierno; Titulaciones profesionales; Ordenamiento jurídico.
\end{abstract}

\begin{abstract}
Previous studies describe a height of outdoor sports and the consequent need to train qualified instructors. Even so, the educational system of instructors of these activities is still being ordered and structured. This article aims to compare the regulations in the professional certifications of winter sports (alpine skiing, cross-country skiing, and snowboarding) and analyze the differences in regulations of the autonomous communities of Spain. The content of the state regulations and the current regulatory development of each autonomous regions regarding professional certifications in winter sport modalities were analyzed. In 2018, eight communities developed specific regulations regarding professional certifications in winter sport modalities, 18 years after the publication of the country regulation. In general, heterogeneity is observed in the regulations between the different communities and also in comparison with the country regulations in aspects as important as professional capacities, exercise of the professional activity, subjects, time distribution, total teaching hours, and proportion of theoretical and practical hours. A homogenization of the regulations of professional certifications in winter sports is necessary among autonomous communities, with the aim of ensuring a convergent line for the professional practice of these modalities throughout the country territory.
\end{abstract}

Keywords: Sports instructors, Superior sports instructors, Outdoor sports, Winter sports, Professional certifications, Legal system.

\section{Introducción}

Más de 20 años atrás, ya se describió el auge adquirido por las actividades físico-deportivas en el medio natural (en adelante, ADMN) y cómo dicho auge comporta «un aumento progresivo de los practicantes y de las estructuras implicadas en este tipo de actividades, un mayor número de profesionales dedicados a este tipo de actividades», entre otras cosas; es decir, comporta «el crecimiento de un gran mercado, el de las ADMN» (Camps, Carretero y Perich, 1995, p.44). La afluencia de practicantes a los entornos naturales, creciente en los últimos años, ha generado una «proliferación de nuevas tendencias, nuevos deportes y diversidad de actividades» (López et al., 2019). El medio natural se ha convertido en un «espacio deportivo abierto a cualquier persona [...], lo que a su vez ha implicado un aumento de accidentes» (Inglés y Seguí, 2012b, p.89). En los últimos años, se han realizado múltiples estudios de datos sobre la siniestralidad en los deportes de montaña, tanto en varias regiones del territorio español (Avellanas, 1995; Mediavilla y Villota, 2012; Nerín y Morandeira, 2005; Sánchez, 2016; Vela, 2009), como

Fecha recepción: 15-05-19. Fecha de aceptación: 14-10-19 Joana Sans Osanz jsans@gencat.cat en diversas áreas de otros países (Powel, 2007; Wild, 2008). Todos estos estudios evidencian el gran crecimiento de siniestros producidos durante la práctica de ADMN. Existe normativa reguladora de la responsabilidad de los agentes implicados en las ADMN, sobre el ámbito profesional y también sobre titulaciones, pero han aumentado las demandas judiciales derivadas de accidentes, hecho que «permite entrever que el ordenamiento jurídico sigue siendo un gran desconocido para el sector» (Inglés y Seguí, 2012b, p. 90). El mencionado incremento de las ADMN, la mayor participación y la accidentalidad, así como el peso que el ámbito deportivo está adquiriendo en la economía del territorio -un 2,4\% del PIB en Cataluña, por ejemplo-, entre otros, han motivado y justificado la necesidad de formar personal cualificado a través de la estructuración y ordenación de un sistema educativo y formativo de profesionales en este ámbito. Se reporta un «actual crecimiento en el número de estudiantes que acceden a cursar enseñanzas deportivas de régimen especial» en España. En el curso 2009/10 había 5250 personas cursando estudios de grado medio y 675 cursando grado superior. En 2014 ese número ya se superó «progresando hacia cifras más elevadas» (Madrera et al., 2014, p.79).

Así, el aumento progresivo de practicantes de ADMN, la necesidad de la formación de los profesionales que las diri- 
gen y conducen, y el creciente número de estudiantes de enseñanzas deportivas, entre otros factores, justifican, por tanto, la urgencia de una evaluación de la situación actual sobre la estructura y ordenación de las formaciones técnicas en ADMN.

Aunque sí existen estudios generales sobre la formación de técnicos/as en el ámbito deportivo (González, 2011; Instituto Nacional de las Cualificaciones y Consejo Superior de Deportes, 2007; Projet Vocasport, 2004), sobre el ordenamiento jurídico en empresas de turismo activo (Bonnet, Wehbe-Herrera y Lobo, 2019; Inglés y Seguí, 2012a; Nasarre, 2000, 2008) y también existen análisis del desarrollo curricular de la Educación Física en la ESO (López-Gil, Devantel, y Renato, 2019; Méndez, Pérez, Méndez, Fernández, y Prieto, 2017), no existen análisis concretos vinculados a identificar los contenidos o competencias específicas en los textos legislativos referentes a las titulaciones de técnicos/as para la conducción de ADMN.

Para abordar la situación de la formación técnica para la conducción de actividades en el medio natural, resulta imprescindible tener en cuenta tres factores: a) el sistema educativo y formativo de profesionales de las ADMN en la enseñanza no-universitaria; b) la ordenación y posterior regulación del espacio profesional y c) la organización competencial de la administración pública (estatal y autonómica).

El sistema educativo y formativo de profesionales de ADMN aún hoy está siendo ordenado y estructurado. Tomando como punto de partida la Ley 10/1990 del Deporte, en el art. 55.1, se encomienda al Gobierno, a propuesta del Ministerio de Educación y Ciencia, la regulación de las enseñanzas de los técnicos deportivos. A este propósito, se sucedieron posteriores leyes y decretos específicos con el fin de establecer los títulos y enseñanzas mínimas (en adelante, EM) de la formación técnica en este ámbito, hasta llegar, en el año 2014, a disponer de seis modalidades reguladas en el marco normativo de la LOGSE, ocho especialidades en el marco normativo de la LOE y aproximadamente 40 planes formativos diversos en el territorio español (Madrera, Garrido y Esteban, 2015, p.153). Dicha integración en el sistema educativo de las titulaciones y enseñanzas de técnico deportivo fue acometida con el Real Decreto 1913/1997, posteriormente derogado por el Real Decreto 1363/2007, de 24 de octubre, por el que se establece la ordenación general de las enseñanzas deportivas de régimen especial (en adelante, EDRE). La pertenencia de España a la Unión Europea conllevó la adaptación de su ordenamiento jurídico a las recomendaciones del Parlamento y el Consejo Europeo (EQFMEC, 2008). Unos años más tarde, se describe la implantación de las EDRE como «poco homogénea e insuficiente a la demanda» porque se rige en «modelos diversos para realidades distintas, por impartirse en diferentes modelos de centros de enseñanza y por basarse en modelos de implantación autonómica diversos» (Madrera, et al., 2014, p.79).

En relación a la ordenación y regulación del espacio profesional, se reporta que la oferta formativa en actividades en el medio natural es «muy variada, aspecto que facilita el acceso a la mayoría de población» pero también «provoca cierta confusión en cuanto a las competencias para las que capacita el aprendizaje de estos contenidos y sus posibilida- des reales para desarrollar una profesión vinculada a las ADMN» (Sáez y Giménez, 2005). La titulación que se requiere para el ejercicio de técnico en $\mathrm{ADMN}$ «es uno de los temas que continúa en vías de resolución» y todavía hoy se continúa «en un periodo de transición, aunque cabe apuntar también que se ha avanzado mucho desde los inicios en 1997» (Inglés y Seguí, 2012a). Es necesario, también, tener en cuenta los datos recogidos por Martínez, Campos, González y Viaño (2012) sobre el porcentaje de personas que trabajan en funciones de actividad física y deporte en instalaciones naturales y turísticas que no poseen titulación alguna: entre el 40 y el 58\% ocupan puestos de trabajo y no son titulados. Estas prácticas físico-deportivas y sus consecuentes estructuras (privadas y públicas) «requieren un ordenamiento funcional y una regulación profesional para preservar la salud de los practicantes y atender los aspectos relacionados con su seguridad» (Martínez, et al., 2012).

En relación a la organización competencial de la administración pública, existen dos administraciones públicas principales que tienen competencias, exclusivas o compartidas, vinculadas al sistema educativo y formativo de profesionales de ADMN en la enseñanza no-universitaria y la ordenación y posterior regulación del espacio profesional: el Estado y las Comunidades Autónomas (en adelante, CCAA). El desarrollo de dichas competencias implica un trabajo colaborativo o coordinado (Madrera, et al., 2014) y, en algunos casos, un ejercicio de coherencia por parte de las administraciones implicadas. Resulta de especial interés conocer qué administración es la competente para saber qué marco normativo debe atenderse en caso de duplicidades normativas, o en caso de contradicciones entre las mismas. En este sentido, años atrás se destacó la «diversidad de poderes públicos implicados en la formación» de estas actividades (Camps, et al., 1995, p.44) y se inició la tendencia, por parte de las CCAA y del Estado, de «regular ese tipo de formaciones, eliminando el monopolio que hasta la fecha tenían las federaciones e intentando introducir todo el conjunto de formaciones profesionalizadas en el campo del deporte dentro del contexto académico reglado» (Camps, et al., 1995, p.50). Años después se describe todavía una ordenación diversa de la regulación del ámbito de las actividades físico-deportivas en el medio natural en el conjunto del estado español y sus CCAA (Inglés y Seguí, 2012a). «La importante vinculación entre titulaciones y formación, por un lado, y ocupaciones y profesiones, por el otro» genera la necesidad de establecimiento de «una ordenación y posterior regulación del espacio profesional basada en las actividades de ocupación propias del deporte» (Madrera, et al., 2015, p.156).

Es a partir de este contexto que el presente artículo pretende, mediante un análisis de la legislación vigente en la materia, analizar y comparar la normativa estatal con el desarrollo normativo de cada CCAA en las titulaciones de técnico deportivo (en adelante, TD) y técnico deportivo superior (en adelante, TDS) en la especialidad de los deportes de invierno en España.

\section{Método}

Se ha realizado un exhaustivo y sistematizado análisis de contenido de los textos que conforman el ordenamiento jurí- 
dico Estatal y de las CCAA en materia de las titulaciones de TD y TDS en las especialidades de Deportes de Invierno (esquí alpino, esquí de fondo y snowboard).

Se detallan, a continuación, los textos normativos analizados:

\section{Marco normativo estatal}

En materia de las titulaciones de deportes de invierno: Real Decreto 319/2000, de 3 de marzo, por el que se establecen los títulos de Técnico Deportivo y Técnico Deportivo superior en las especialidades de los Deportes de Invierno, se aprueban las correspondientes enseñanzas mínimas y se regulan las pruebas de acceso a estas enseñanzas (en adelante, RD319/2000)

Desarrollo normativo correspondiente a cada CCAA

Aragón: Orden de 14 de diciembre de 2001, del Departamento de Educación y Ciencia, por la que se establecen, con carácter experimental, los currículos y las pruebas de acceso específicas correspondientes a los títulos de técnico deportivo y técnico deportivo superior de las especialidades de los deportes de invierno en la Comunidad Autónoma de Aragón

Cataluña: Decreto 281/2002, de 5 de noviembre, por el que se establecen los currículos y se regulan las pruebas de acceso específicas de los títulos de técnico deportivo en las disciplinas de los deportes de invierno siguientes: esquí alpino, esquí de fondo y snowboard y Departament d'Ensenyament, Generalitat de Catalunya (2018). Documentos para la organización y la gestión de los centros. Ciclos deportivos LOE y LOGSE.

Comunidad de Madrid: Orden 5094/2003, de 4 de septiembre, del Consejero de Educación por la que se establecen los currículos y las pruebas de acceso correspondientes a las enseñanzas conducentes a los títulos de Técnico Deportivo y de Técnico Deportivo Superior de las especialidades de los Deportes de Invierno, y Orden 3694/2009, de 28 de julio, por la que se establece para la Comunidad de Madrid la distribución horaria de las Enseñanzas Deportivas de régimen especial de las modalidades de Atletismo, Baloncesto, Balonmano, Deportes de Invierno, Deportes de Montaña y Escalada y Fútbol

Andalucía: Decreto 197/2005, de 13 de septiembre, por el que se establecen los requisitos y pruebas específicas de acceso correspondientes a los títulos de Técnico Deportivo y de Técnico Deportivo Superior de las especialidades de los Deportes de Invierno

Principado de Asturias: Decreto 29/2007, de 29 de marzo, por el que se establecen los currículos y se regulan las pruebas y requisitos de acceso específicos correspondientes a los Títulos de Técnico Deportivo y de Técnico Deportivo Superior en las especialidades de los Deportes de Invierno en el Principado de Asturias, y Decreto 30/2004, de 1 de abril, por el que se establece la ordenación general de las enseñanzas de régimen especial correspondientes a los títulos de Técnico Deportivo y de Técnico Deportivo Superior en el Principado de Asturias

País Vasco: Decreto 172/2010, de 29 de junio, por el que se establece el currículo correspondiente a las enseñanzas de Técnico Deportivo y Técnico Deportivo Supe- rior en las especialidades de los Deportes de Invierno y se regulan las pruebas y requisitos de acceso correspondientes

Cantabria: Orden EDU/57/2010, de 7 de julio, de los currículos y las pruebas de carácter específico de acceso correspondientes a los títulos de Técnico Deportivo y Técnico Deportivo Superior en las especialidades de Deportes de Invierno en la Comunidad Autónoma de Cantabria

Castilla y León: Decreto 64/2010, de 30 de diciembre, por el que se establecen los currículos y se regula la prueba de acceso de carácter específico correspondiente a los títulos de Técnico Deportivo y Técnico Deportivo Superior de las Especialidades de los Deportes de Invierno en la Comunidad de Castilla y León

Islas Baleares, Islas Canarias, Castilla la Mancha, Extremadura, Galicia, Comunidad Valenciana, Región de Murcia, Comunidad Foral de Navarra y La Rioja: no han desarrollado normativa específica hasta el momento del análisis.

\section{Resultados}

Unidades de competencia, capacidades profesionales, perfil profesional y ubicación en el ámbito deportivo

Todas las CCAA excepto Cataluña, Euskadi y Andalucía citan textualmente las unidades de competencia y las capacidades profesionales que aparecen en el RD319/2000 para cada uno de los niveles de formación.

Cataluña es la CCAA que más diferencias presenta en cuanto al contenido. La norma estatal establece tres unidades de competencia en todos los niveles y modalidades mientras que en el técnico deportivo de primer nivel (en adelante, TDN1) de las tres modalidades, Cataluña establece cuatro, añadiendo: «Promover la realización de actividades» de cada especialidad, y especifica cuatro capacidades profesionales dentro de esta unidad de competencia que no aparecen en la norma estatal. También en el TDN1 de las tres modalidades, Cataluña añade la competencia sobre enseñar y hacer cumplir las normas básicas del reglamento, la cual no aparece en la norma estatal. En el técnico deportivo de segundo nivel (en adelante, TDN2) de las tres modalidades, Cataluña establece ocho capacidades profesionales menos que la norma estatal y, en el caso del TDN2 de esquí de fondo, Cataluña especifica que se refiere a «esquí de fondo y otras actividades nórdicas» (Decreto 281/2002: Anexo 2, 3.2), mientras que en la norma estatal solo se refiere al esquí de fondo; no se concreta a qué otras actividades nórdicas se refiere.

Euskadi incluye cuatro unidades de competencia (y únicamente en el TDN1 de esquí alpino) añadiendo una a las establecidas en el RD319/2000: «Asegurarse de que la actividad profesional siempre se desarrolle en términos de tolerancia e igualdad entre hombres y mujeres» (Decreto 172/2010: Anexo 2, 1.2).

Euskadi y Andalucía no se diferencian en el contenido sino únicamente en el uso del lenguaje y el género: son las únicas CCAA que no usan el masculino genérico y optan por usar otras opciones (por ejemplo «los y las deportistas», «la persona enferma», «personas deportistas»). Tanto el RD319/2000 como las demás CCAA utilizan únicamente el masculino genérico. 


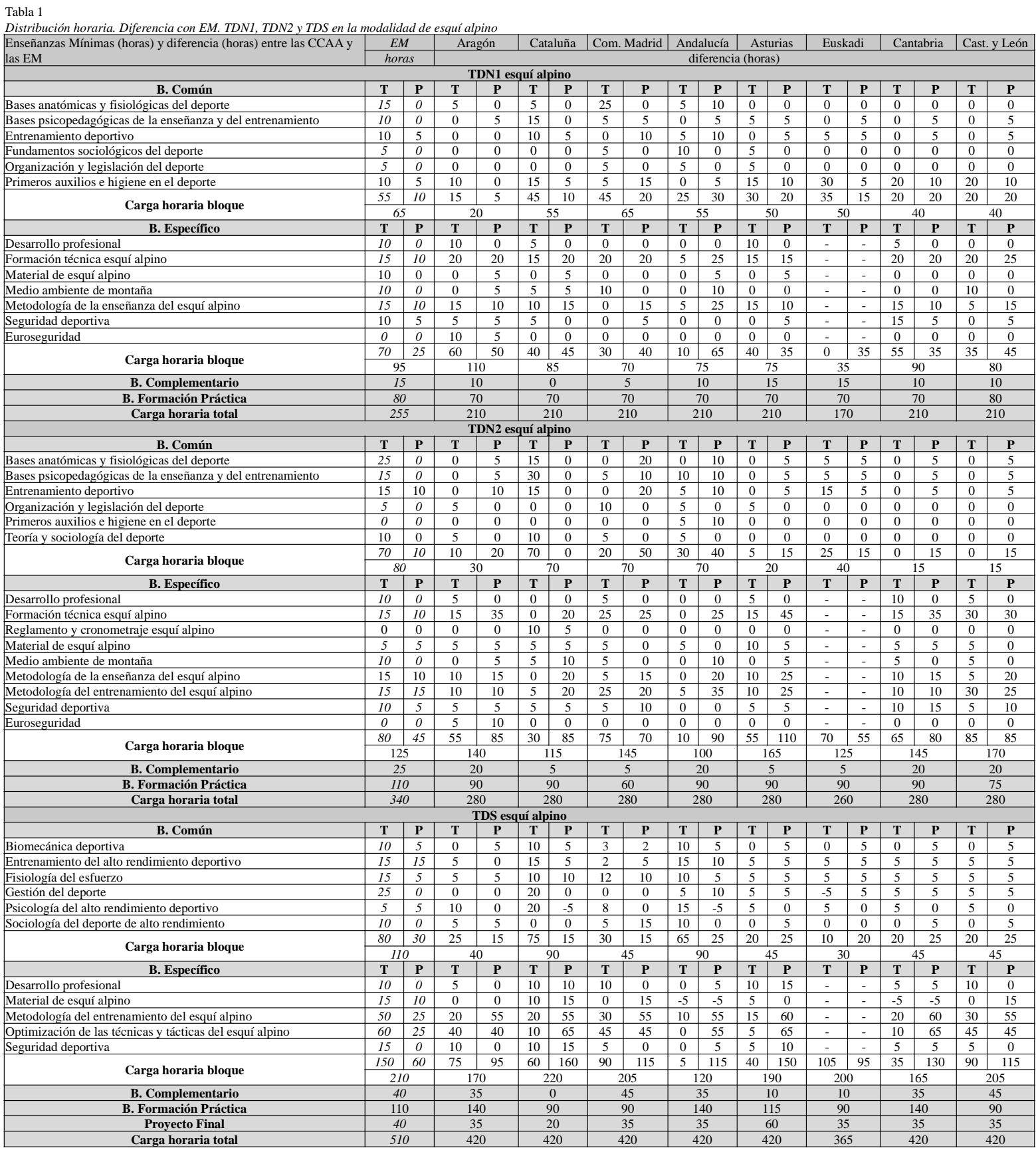

En relación al perfil profesional y ubicación en el ámbito deportivo, no existe una gran divergencia entre los desarrollos normativos de las CCAA, más bien existe una concreción y ampliación de lo expuesto en el RD319/2000 por parte de Cataluña (en el caso de TDN1 y TDN2 de todas las modalidades) y Andalucía (en el caso del TDN1 de esquí alpino).

Por lo que se refiere al ejercicio de la actividad profesional, alguien con el certificado de TDN1 de esquí alpino ejercerá «en el ámbito de la iniciación deportiva, sesiones de enseñanza de esquiadores, en las pistas balizadas de una estación de deportes de invierno. Se excluye expresamente la enseñanza del esquí de montaña y del esquí de fondo» (RD319/2000: Anexo III, 2.4). «Este técnico actuará bajo la supervisión general de técnicos y/o profesionales de nivel superior al suyo» (RD319/2000: Anexo III, 2.5). En este punto, la normativa de Andalucía añade y clarifica que el Snowboard se excluye también del ámbito de actuación del
TDN1 de Esquí Alpino (Decreto 197/2005: Anexo I, 2.) Del mismo modo, la normativa de Cataluña añade y clarifica que la práctica del Snowboard también se excluye de su ámbito de actuación (Decreto 281/2002: Anexo 2, apartado de primer nivel de técnico de deporte en esquí alpino, 3.3.1). Ésta CCAA también añade: «Principales ocupaciones que se pueden desarrollar cuando se haya adquirido la competencia profesional definida en el perfil: promotor de actividades de deportes de invierno, monitor/instructor de esquí alpino» (Decreto 281/2002: Anexo 2, apartado de primer nivel de técnico de deporte en esquí alpino, 3.3.1).

Un TDN2 de esquí alpino tiene competencias en «enseñanza y entrenamiento del esquí alpino. Su actuación profesional se realizará en pistas balizadas y fuera de pista en el dominio esquiable de una estación de deportes de invierno» (RD319/2000: Anexo III, 4.4). También deja claro que se excluye de su actuación el «esquí de montaña y la conducción 
Tabla 2

Distribución horaria. Diferencia con EM. TDN1, TDN2 y TDS en la modalidad de esquí de fondo

\begin{tabular}{|l|c|c|c|c|c|c|c|c|c|c|c|}
\hline Enseñanzas Mínimas (horas) y diferencia (horas) entre las CCAA y las & $E M$ & Aragón & Cataluña & Com. Madrid & Andalucía & Asturias & Euskadi & Cantabria & Cast. y León \\
\hline
\end{tabular}

EM

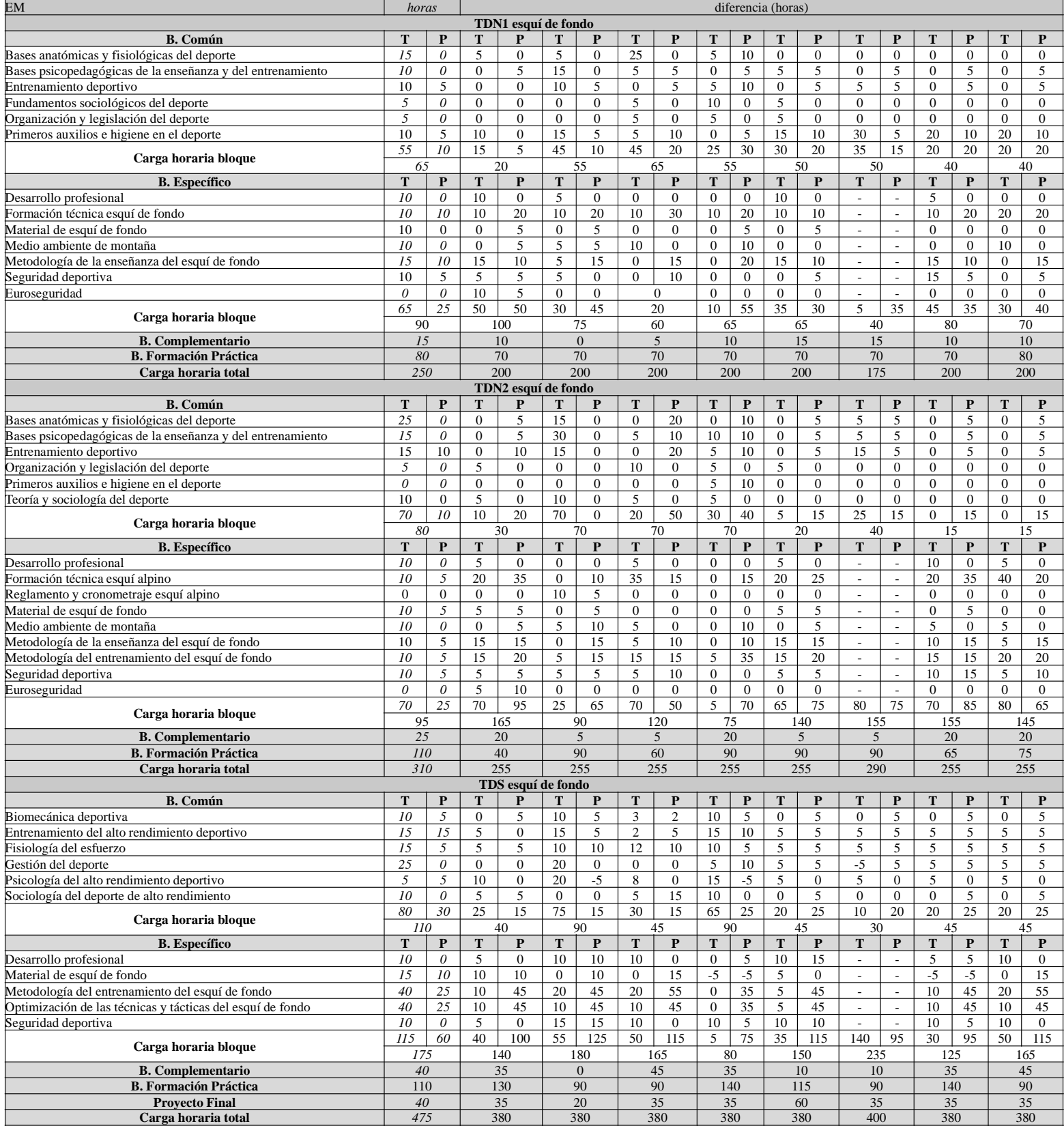

de individuos o grupos por terrenos que necesiten para la progresión técnicas y materiales específicos de escalada o alpinismo» (RD319/2000: Anexo III, 4.4). Cataluña añade «Esquí de Fondo y Snowboard» como actividades excluidas de su ámbito de actuación (Decreto 281/2002: Anexo 2, apartado de segundo nivel de técnico de deporte en esquí alpino, 3.3.1). También añade las principales ocupaciones que se pueden desarrollar una vez adquirida la competencia profesional que se define en el perfil y éstas son «profesor de esquí alpino, entrenador de esquí alpino, director de actividades de esquí alpino, cronometrador de competiciones de esquí alpino» (Decreto 281/2002: Anexo 2, apartado de segundo nivel de técnico de deporte en esquí alpino, 3.3.1).

El certificado de TDN1 de esquí de fondo capacita para la «iniciación deportiva, desarrollando sesiones de enseñanza de esquiadores, en pistas balizadas de una estación de deportes de invierno» (RD319/2000: Anexo IV, 2.4). Queda ex- cluida de su ámbito de actuación «la enseñanza del esquí de montaña y del esquí alpino» (RD319/2000: Anexo IV, 2.4). «Este técnico actuará bajo la supervisión general de técnicos y/o profesionales de nivel superior al suyo» (RD319/ 2000: Anexo IV, 2.5). Cataluña también añade ocupaciones: «promotor de actividades de deportes de invierno, monitor/ instructor de esquí de fondo» (Decreto 281/2002: Anexo 3, apartado de primer nivel de técnico de deporte en esquí de fondo, 3.3.1).

Un TDN2 de esquí de fondo tiene competencias en el «ámbito de la enseñanza y del entrenamiento del esquí de fondo. Su actuación profesional se realizará en pistas balizadas. Se excluye expresamente de su ámbito de actuación la enseñanza del esquí alpino y del esquí de montaña» (RD319/2000: Anexo IV, 4.4). Cataluña añade que tiene competencias en «enseñanza y entrenamiento de otras actividades nórdicas», aunque no se concretan qué otras activida- 
Tabla 3

Distribución horaria. Diferencia con EM. TDN1, TDN2 y TDS en la modalidad de snowboard

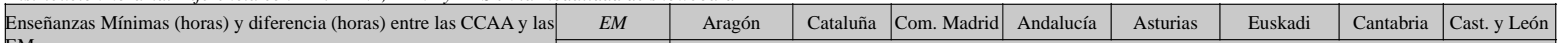

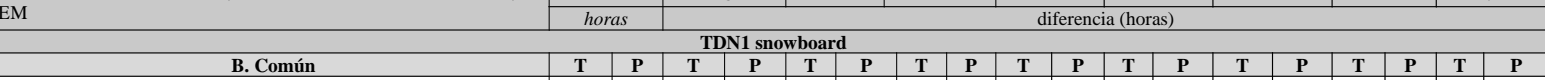

\begin{tabular}{|l|c|c|c|c|c|c|c|c|c|c|c|c|c|c|c|c|c|c|c|}
\multicolumn{1}{|c|}{ B. Común } & T & $\mathbf{P}$ & $\mathbf{T}$ & $\mathbf{P}$ & $\mathbf{T}$ & $\mathbf{P}$ & $\mathbf{T}$ & $\mathbf{P}$ & $\mathbf{T}$ & $\mathbf{P}$ & $\mathbf{T}$ & $\mathbf{P}$ & $\mathbf{T}$ & $\mathbf{P}$ & $\mathbf{T}$ & $\mathbf{P}$ & $\mathbf{T}$ & $\mathbf{P}$ \\
\hline Bases anatómicas y fisiológicas del deporte & 15 & 0 & 5 & 0 & 5 & 0 & 25 & 0 & 5 & 10 & 0 & 0 & 0 & 0 & 0 & 0 & 0 & 0 \\
\hline Bases psicopedagógicas de la enseñanza y del entrenamiento & 10 & 0 & 0 & 5 & 15 & 0 & 5 & 5 & 0 & 5 & 5 & 5 & 0 & 5 & 0 & 5 & 0 & 5 \\
\hline
\end{tabular}

\begin{tabular}{|l|c|c|c|c|c|c|c|c|c|c|c|c|c|c|c|c|c|c|}
\hline Bases psicopedagógicas de la enseñanza y del entrenamiento & 10 & 0 & 0 & 5 & 15 & 0 & 5 & 5 & 0 & 5 & 5 & 5 & 0 & 5 & 0 & 5 & 0 & 5 \\
\hline Entrenamiento deportivo & 10 & 5 & 0 & 0 & 10 & 5 & 0 & 10 & 5 & 10 & 0 & 5 & 5 & 5 & 0 & 5 & 0 & 5 \\
\hline Fundamentos sociológicos del deporte & 5 & 0 & 0 & 0 & 0 & 0 & 5 & 0 & 10 & 0 & 5 & 0 & 0 & 0 & 0 & 0 & 0 & 0 \\
\hline
\end{tabular}

Organización y legislación del deporte

\begin{tabular}{|c|c|c|c|c|c|c|c|c|c|c|c|c|c|c|c|c|c|}
5 & 0 & 0 & 0 & 0 & 5 & 0 & 5 & 0 & 5 & 0 & 0 & 0 & 0 & 0 & 0 & 0 \\
\hline 10 & 5 & 10 & 0 & 15 & 5 & 5 & 15 & 0 & 5 & 15 & 10 & 30 & 5 & 20 & 10 & 20 & 10 \\
\hline
\end{tabular}

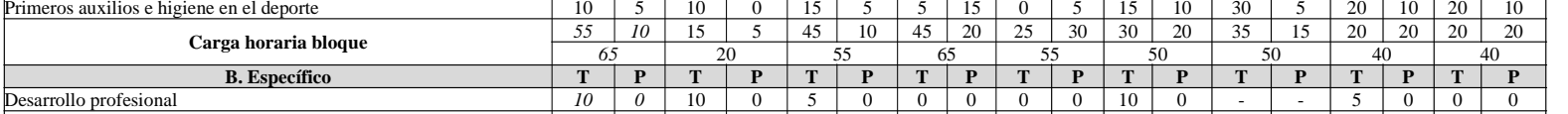

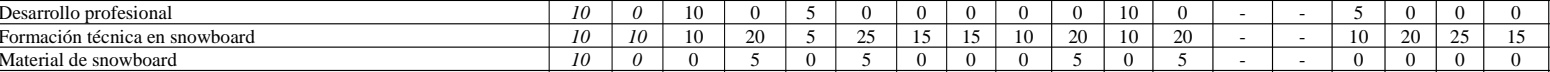

Medio ambiente de moñ

Metodología de la enseñanza del snowboard

Metodología del entrenamiento del snowboard

Seguridad deportiva

Euroseguridad

Carga horaria bloque

B. Complementario

B. Formación Práctica

Carga horaria total

\begin{tabular}{|c|c|c|c|c|c|c|}
\hline B. Formación Práctica & \multicolumn{2}{c|}{80} & 70 & 70 \\
\hline Carga horaria total & 245 & \multicolumn{2}{|c|}{205} & 205 \\
\hline \multicolumn{7}{|c|}{ TDN2 snowboard } \\
\hline B. Común & T & P & T & P & T & P \\
\hline
\end{tabular}

\begin{tabular}{|l|c|c|c|c|c|c|c|}
\hline Bases anatómicas y fisiológicas del deporte & 25 & 0 & 0 & 5 & 15 & \\
\hline Bases psicopedagógicas de la enseñanza y del entrenamiento & 15 & 0 & 0 & 5 & 30 & \\
\hline Entrenamiento deportivo & 15 & 10 & 0 & 10 & 15 & 0
\end{tabular}

Entrenamiento deportivo

Primeros auxilios e higiene en el deporte

Teoría y sociología del deporte

\begin{tabular}{|c|c|c|c|c|c|c|c|c|c|c|c|c|c|c|c|c|c|c|}
\hline \multirow[b]{2}{*}{ Carga horaria bloque } & \multirow{2}{*}{\multicolumn{2}{|c|}{$\begin{array}{c}70 \mid 10 \\
80\end{array}$}} & 10 & 20 & 70 & 0 & 20 & 50 & 30 & 40 & 5 & 15 & 25 & 15 & 0 & 15 & 0 & 15 \\
\hline & & & \multicolumn{2}{|c|}{30} & \multicolumn{2}{|c|}{70} & \multicolumn{2}{|c|}{70} & \multicolumn{2}{|c|}{70} & \multicolumn{2}{|c|}{20} & \multicolumn{2}{|c|}{40} & \multicolumn{2}{|c|}{15} & \multicolumn{2}{|c|}{15} \\
\hline B. Específico & $\mathbf{T}$ & $\mathbf{P}$ & $\mathbf{T}$ & $\mathbf{P}$ & $\mathbf{T}$ & $\mathbf{P}$ & $\mathbf{T}$ & $\mathbf{P}$ & $\mathbf{T}$ & $\mathbf{P}$ & $\mathbf{T}$ & $\mathbf{P}$ & $\mathbf{T}$ & $\mathbf{P}$ & $\mathbf{T}$ & $\mathbf{P}$ & $\mathbf{T}$ & $\mathbf{P}$ \\
\hline Formación técnica snowboard & 10 & 10 & 20 & 35 & 5 & 10 & 20 & 20 & 5 & 15 & 20 & 40 & - & - & 20 & 35 & 25 & 25 \\
\hline Reglamento y cronometraje del snowboard & 0 & 0 & 0 & 0 & 10 & 5 & 0 & 0 & 0 & 0 & 0 & 0 & - & - & 0 & 0 & 0 & 0 \\
\hline Medio ambiente montaña & 10 & 0 & 0 & 5 & 5 & 10 & 5 & 0 & 0 & 10 & 0 & 5 & - & - & 5 & 0 & 5 & 0 \\
\hline \multirow[t]{2}{*}{ Metodología enseñanza snowboard } & 10 & 10 & 15 & 10 & 5 & 15 & 15 & 15 & 5 & 15 & 15 & 10 & - & - & 10 & 10 & 20 & 15 \\
\hline & 10 & 10 & 15 & 15 & 10 & 15 & 20 & 20 & 10 & 30 & 20 & 20 & - & - & 15 & 10 & 25 & 25 \\
\hline Seguridad deportiva & 10 & 5 & 5 & 5 & 5 & 5 & 5 & 10 & 0 & 0 & 5 & 5 & - & - & 10 & 15 & 5 & 10 \\
\hline B. Complementario & \multicolumn{2}{|c|}{25} & \multicolumn{2}{|c|}{$\begin{array}{c}155 \\
20\end{array}$} & \multicolumn{2}{|c|}{$\begin{array}{c}105 \\
5\end{array}$} & \multicolumn{2}{|c|}{$\begin{array}{c}135 \\
5\end{array}$} & \multicolumn{2}{|c|}{$\begin{array}{l}90 \\
20\end{array}$} & \multicolumn{2}{|c|}{5} & \multicolumn{2}{|c|}{$\frac{140}{5}$} & \multicolumn{2}{|c|}{20} & \multicolumn{2}{|c|}{20} \\
\hline B. Formación Práctica & \multicolumn{2}{|c|}{110} & \multicolumn{2}{|c|}{65} & \multicolumn{2}{|c|}{90} & \multicolumn{2}{|c|}{60} & \multirow{2}{*}{\multicolumn{2}{|c|}{90}} & \multirow{2}{*}{\multicolumn{2}{|c|}{90}} & \multirow{2}{*}{\multicolumn{2}{|c|}{90}} & \multirow{2}{*}{\multicolumn{2}{|c|}{90}} & \multicolumn{2}{|c|}{75} \\
\hline Carga horaria total & & & & & & & & & & & & & & & & & & 70 \\
\hline & & & & S sne & boar & & & & & & & & & & & & & \\
\hline B. Común & $\mathbf{T}$ & $\mathbf{P}$ & $\mathbf{T}$ & $\mathbf{P}$ & $\mathbf{T}$ & $\mathbf{P}$ & $\mathbf{T}$ & $\mathbf{P}$ & $\mathbf{T}$ & $\mathbf{P}$ & $\mathbf{T}$ & $\mathbf{P}$ & $\mathbf{T}$ & $\mathbf{P}$ & $T$ & $\mathbf{P}$ & $\mathbf{T}$ & $\mathbf{P}$ \\
\hline
\end{tabular}

Biomecánica deportiva

Entrenamiento del alto rendimiento deportivo

Fisiología del esfuerzo

Psicología del alto rendimiento deportivo

Sociología del deporte de alto rendimiento

\begin{tabular}{|c|c|c|c|c|c|c|c|c|c|c|c|c|c|c|c|c|c|c|}
\hline \multirow{2}{*}{ Carga horaria bloque } & \multirow{2}{*}{\multicolumn{2}{|c|}{$\frac{80 \mid 30}{110}$}} & \multirow{2}{*}{\multicolumn{2}{|c|}{$\begin{array}{l}25 \quad 15 \\
40\end{array}$}} & \multirow{2}{*}{\multicolumn{2}{|c|}{90}} & \multirow{2}{*}{\multicolumn{2}{|c|}{45}} & \multirow{2}{*}{\multicolumn{2}{|c|}{90}} & \multirow{2}{*}{\multicolumn{2}{|c|}{$\begin{array}{l}20 \mid 25 \\
45\end{array}$}} & \multicolumn{2}{|c|}{$10 \mid 20$} & \multicolumn{2}{|c|}{20125} & \multirow{2}{*}{\multicolumn{2}{|c|}{20120}} \\
\hline & & & & & & & & & & & & & & & & & & \\
\hline Desarrollo profesional & 10 & 0 & 5 & 0 & 10 & 10 & 10 & 0 & 0 & 5 & 10 & 15 & - & - & 5 & 5 & 10 & 0 \\
\hline Material de snowboard & 10 & 10 & 5 & 0 & 5 & 10 & 5 & 15 & 0 & -5 & 10 & 0 & - & - & 0 & -5 & 5 & 15 \\
\hline Metodología del entrenamiento del snowboard & 30 & 10 & 40 & 70 & 10 & 50 & 25 & 15 & -10 & 40 & 0 & 40 & - & - & 20 & 30 & 25 & 15 \\
\hline Seguridad deportiva & 10 & 0 & 15 & 0 & 15 & 15 & 10 & 0 & 10 & 5 & 10 & 10 & - & - & 10 & 5 & 10 & 0 \\
\hline \multirow{2}{*}{ Carga horaria bloque } & 100 & 35 & 125 & 120 & 40 & 120 & 80 & 65 & -20 & 80 & 30 & 100 & 155 & 120 & 45 & 60 & 80 & 65 \\
\hline & \multicolumn{2}{|c|}{135} & \multicolumn{2}{|c|}{245} & \multicolumn{2}{|c|}{160} & \multicolumn{2}{|c|}{145} & \multicolumn{2}{|c|}{60} & \multicolumn{2}{|c|}{130} & \multicolumn{2}{|c|}{275} & \multicolumn{2}{|c|}{105} & \multicolumn{2}{|c|}{145} \\
\hline B. Complementario & \multicolumn{2}{|c|}{40} & \multicolumn{2}{|c|}{$\frac{35}{5}$} & \multicolumn{2}{|c|}{$\frac{0}{90}$} & \multicolumn{2}{|c|}{$\begin{array}{l}45 \\
90\end{array}$} & \multicolumn{2}{|c|}{$\frac{35}{140}$} & \multicolumn{2}{|c|}{$\frac{10}{115}$} & \multicolumn{2}{|c|}{$\frac{10}{90}$} & \multicolumn{2}{|c|}{$\frac{35}{140}$} & \multicolumn{2}{|c|}{45} \\
\hline
\end{tabular}

des nórdicas. También clarifica que se excluye de su ámbito de actuación la enseñanza del «snowboard y la conducción de personas o grupos por terrenos que requieran de la utilización de técnicas y materiales específicos de escalada o alpinismo». Del mismo modo que en el caso del esquí alpino, añade las ocupaciones siguientes: «profesor de esquí de fondo, entrenador de esquí de fondo, director de actividades de esquí de fondo y otras actividades nórdicas, cronometrador en competiciones de esquí de fondo» (Decreto 281/2002: Anexo 3, apartado de segundo nivel de técnico de deporte en esquí de fondo, 3.3.1).

Las competencias correspondientes al TDN1 se dan en el «ámbito de la iniciación deportiva, desarrollando sesiones de enseñanza del snowboard, en las pistas balizadas de una estación de deportes de invierno» (RD319/2000: Anexo V, 2.4). «Este técnico actuará bajo la supervisión general de técnicos y/o profesionales de nivel superior al suyo» (RD319/
2000: Anexo V, 2.5). Cataluña añade que «se excluye de su ámbito de actuación la enseñanza del esquí de montaña, esquí alpino y esquí de fondo». También clarifica las «principales ocupaciones que se pueden desarrollar cuando se haya adquirido la competencia profesional definida en el perfil: Promotor de actividades de deportes de invierno, Monitor/ Instructor de snowboard» (Decreto 281/2002: Anexo 4, apartado de primer nivel de técnico de deporte en snowboard, 3.3.1). Un TDN2 de Snowboard tiene competencias en la «enseñanza y entrenamiento del snowboard. Su actuación profesional se realizará en pistas balizadas y fuera de pista en el dominio esquiable de una estación de deportes de invierno» (RD319/2000: Anexo V, 4.4). De su ámbito de actuación se excluye expresamente toda actividad en «terrenos que necesiten para la progresión técnicas y materiales específicos de escalada o alpinismo» (RD319/2000: Anexo V, 4.4). Cataluña clarifica que «se excluye de su ámbito de actuación 
la enseñanza del esquí de montaña, esquí alpino y esquí de fondo» y concreta las ocupaciones que se podrán desarrollar y éstas son: «profesor de esquí de snowboard, entrenador de snowboard, director de actividades de snowboard, juez o cabeza de jueces en competiciones de snowboard» (Decreto 281/2002: Anexo 4, apartado de segundo nivel de técnico de deporte en snowboard, 3.3.1).

Todas las normativas coinciden con lo expuesto en el RD319/2000 por lo que se refiere a la tipología de entidades y empresas donde ejercer la profesión. Así, todos los certificados de TDN1 podrán actuar «siempre en el seno de un organismo público o privado relacionado con la práctica». Podrán desarrollar sus funciones en «escuelas deportivas, centros de iniciación deportiva, clubes o asociaciones deportivas, federaciones deportivas, patronatos deportivos, empresas de servicios deportivos, centros escolares (actividades extraescolares)» (RD319/2000: Anexo III, 2.4, Anexo IV, 2.4 y Anexo V, 2.4). Del mismo modo, todos los TDN2 podrán «asumir sus responsabilidades de forma autónoma o en el seno de un organismo público o empresa privada». Podrán desarrollar sus funciones en «escuelas deportivas, clubes o asociaciones deportivas, federaciones deportivas, patronatos deportivos, empresas de servicios deportivos, centros escolares (actividades extraescolares)» (RD319/2000: Anexo III, 4.4, Anexo IV, 4.4 y Anexo V, 4.4). Los TDS podrán «asumir sus responsabilidades de forma autónoma o en el seno de un organismo público o empresa privada». Podrán desarrollar sus funciones en: «centros de alto rendimiento deportivo, centros de tecnificación deportiva, escuelas de deportes de invierno, clubes o asociaciones deportivas, federaciones, patronatos deportivos, empresas de servicios deportivos, centros de formación de técnicos deportivos de deportes de invierno» (RD319/2000: Anexo III, 7.4, Anexo IV, 7.4 y Anexo V, 7.4).

Asignaturas de las formaciones de TDN1, TDN2 y TDS

Estructuralmente, cada nivel formativo se divide en bloques: bloque común (en adelante, BC), bloque específico (en adelante, BE), bloque complementario (en adelante, BComp) y bloque de formación práctica (en adelante, BFP). Además, en las titulaciones de TDS, se añade un bloque: el Proyecto Final.

Durante el análisis de los textos legislativos que establecen los currículos de los títulos de TDN1 y TDN2 de las tres modalidades encontramos que Aragón es la única CCAA que cursa la asignatura de «Euroseguridad» y la incluye en el BE. Dicha materia no aparece en el RD319/2000.

Únicamente Cantabria cursa «Metodología del entrenamiento» en la formación de TDN1 y sólo en la modalidad de Snowboard, mientras que las otras CCAA la cursan a partir del TDN2 para todas las modalidades.

En las formaciones de TDN2, únicamente Cataluña incluye en el BE la asignatura de «Reglamento y cronometraje» de cada modalidad. Dicha materia no aparece en el RD319/ 2000. El hecho de cursar esta asignatura justifica que Cataluña haya establecido ser cronometrador en competiciones de la modalidad como una de las principales ocupaciones de estos profesionales.

En las formaciones de TDN2 de las tres modalidades únicamente Andalucía cursa la asignatura de «Primeros auxi- lios e higiene en el deporte» en el BC, además de cursarla en el TDN1. El resto de CCAA sólo la cursan en el TDN1.

Se muestra homogeneidad en las titulaciones de TDS de las tres modalidades: se fijan las mismas las asignaturas en todas las CCAA y en coincidencia con RD319/2000.

En las formaciones de todos los niveles de las tres modalidades Euskadi no concreta qué asignaturas forman parte del BE, únicamente establece horas teóricas y prácticas totales.

Distribución horaria por asignaturas del desarrollo normativo de cada CCAA y diferencia entre ésta y las EM del RD319/2000

Se puede observar heterogeneidad en la distribución horaria que han establecido las diferentes CCAA para una misma formación. Para evidenciar este hecho, se ha usado la diferencia, por asignaturas y bloques, entre las horas establecidas en el desarrollo normativo de cada CCAA y las horas establecidas en las EM del RD319/2000 (ver Tabla 1, Tabla 2 y Tabla 3).

Cabe destacar el hecho que Euskadi es la única CCAA que ha igualado la carga lectiva total de las tres modalidades, de manera que todos los TDN1 cursan un total de 425 horas, los TDN2 un total de 600 horas y los TDS, 875 horas. En las otras CCAA, ésta carga varía en función de cada modalidad, ajustándose entonces a lo establecido en el RD319/2000.

A pesar de la dicha heterogeneidad, se observa alguna tendencia de grupos.

En el análisis de las formaciones de TDN1 cabe destacar que Castilla y León es la CCAA que más horas dedica al BFP en todas las modalidades. Euskadi sobrepasa en 30h las EM en las tres modalidades con la asignatura de «Primeros auxilios e higiene en el deporte» siendo ésta la mayor diferencia en el BC de todas las CCAA. Aragón apuesta por una formación de carácter más específico dedicando más horas al BE que las otras CCAA. Andalucía es la CCAA que más horas prácticas establece en el BC y el BE.

Los resultados de las formaciones de TDN2 muestran que Castilla y León (en esquí alpino y snowboard) y Aragón (esquí de fondo) apuestan por una formación de carácter más específico dedicando al BE un total de horas mayor que las otras CCAA. Siguiendo el análisis del BE, Asturias es la CCAA que establece una diferencia de carga horaria práctica mayor ( 45 y 40 horas por encima de las EM) en la asignatura de «formación técnica» de esquí alpino y snowboard. En cambio, para la modalidad de esquí de fondo es Castilla y León la que destaca por superar en 40 horas teóricas las EM para la misma asignatura. Centrando la atención en el BC, las CCAA que presentan mayores diferencias por encima de las EM son Cataluña y la Comunidad de Madrid en las tres modalidades. En cuanto a la carga lectiva otorgada al BFP, la mayoría de CCAA destacan por dedicar 90 horas por encima de las EM. Son excepción y, por tanto, dedican un menor volumen horario al BFP las siguientes CCAA: Comunidad de Madrid, Castilla y León (en las tres modalidades), Aragón (en esquí de fondo y snowboard) y Cantabria (esquí de fondo).

En las formaciones de TDS, el análisis del BC establece que Cataluña y Andalucía presentan una mayor diferencia con las EM en comparación con las otras CCAA. Euskadi 
Tabla 4

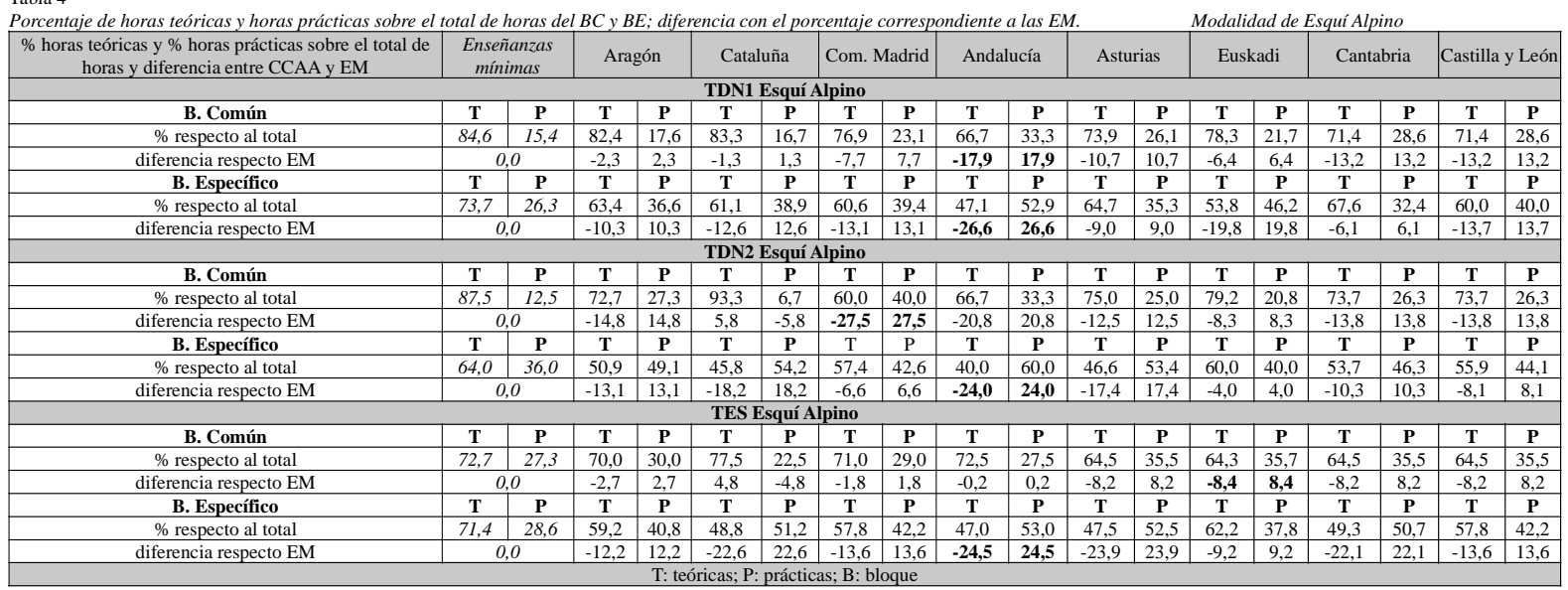

Tabla 5

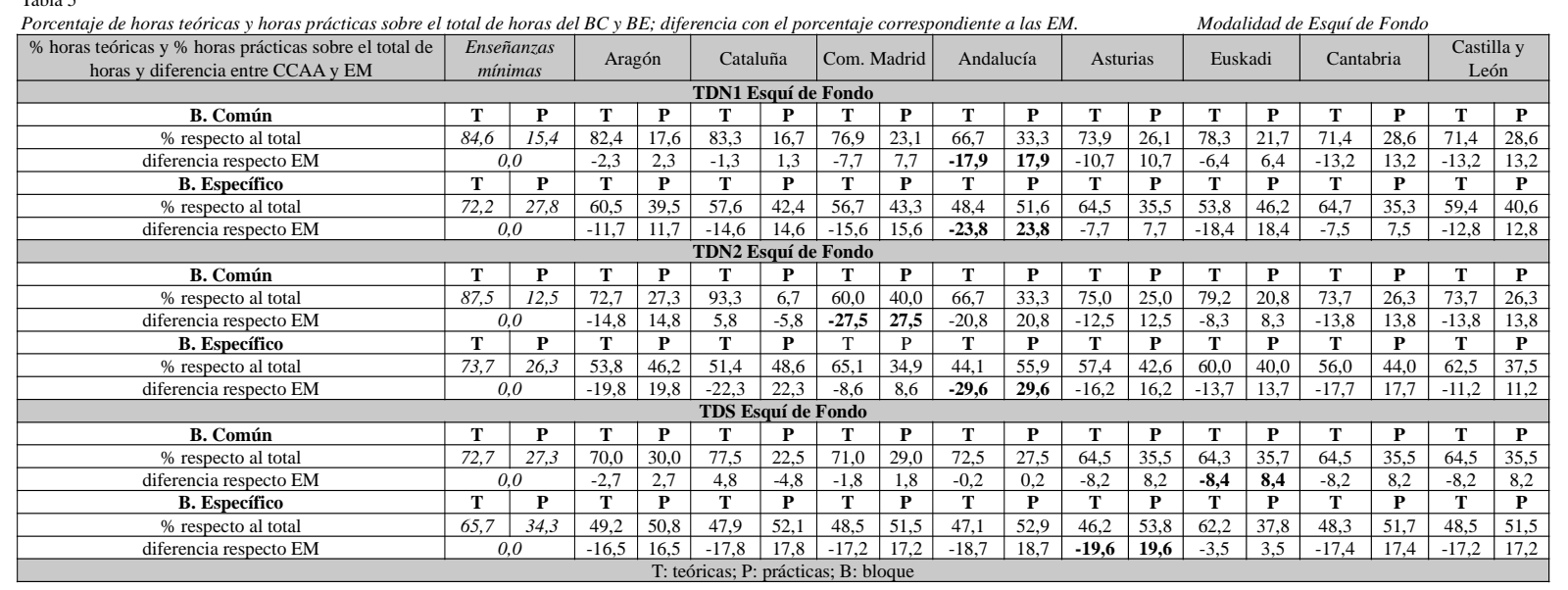

Tabla 6

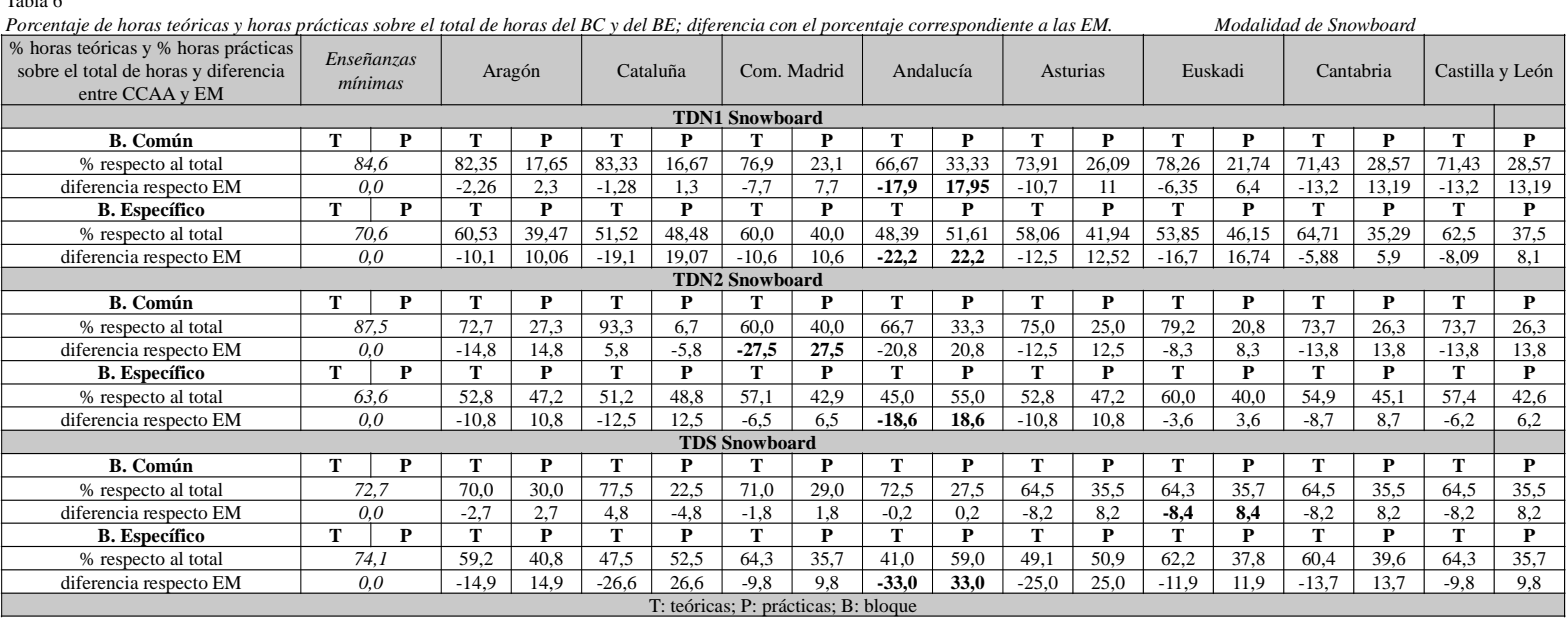

apuesta por dedicar más carga horaria al BE que las otras CCAA en esquí de fondo (diferencia de 235h) y snowboard (diferencia de 275h). En la modalidad de esquí alpino es Cataluña quien destaca (220h). Por lo que se refiere al BFP, Andalucía y Cantabria son las que mayores diferencias presentan con las EM en las tres modalidades. Aragón también destaca en esquí alpino y de fondo. Asturias destaca por dedicar la mayor carga lectiva (60h por encima de EM) al Proyecto Final en las tres modalidades.

Los resultados del análisis del BComp revelan que Cataluña es la CCAA que menos carga horaria dedica a este bloque analizando de manera global las tres modalidades i los tres niveles de formación.

En la Tabla 1, Tabla 2 y Tabla 3 se puede observar como existen CCAA que han establecido una distribución horaria que se queda por debajo de las enseñanzas mínimas (datos negativos en las tablas) dictadas en el RD319/2000. Cabe destacar que ninguna CCAA se ha quedado por debajo de las EM en las formaciones de TDN2 de las tres modalidades, pero sí lo han hecho en el TDN1 y en el TDS. Por ejemplo, Cantabria establece 10 horas por debajo de las EM en las asignaturas «Medio ambiente de montaña» y «Metodología de la enseñanza» en el BE del TDN1 de snowboard. En el BC del TDS de las tres modalidades, Cataluña, Andalucía y 
Euskadi establecen 5 horas por debajo de las EM en las asignaturas de «Gestión del deporte» y «Psicología del alto rendimiento deportivo». En el BE del TDS de esquí alpino y de fondo, Andalucía y Cantabria cursan 5 horas por debajo de las EM en «Material de esquí». Andalucía no llega a las EM en cuatro asignaturas del BE del TDS de snowboard, del mismo modo que Cantabria en «Material de snowboard».

\section{Distribución de horas teóricas y prácticas}

En las Tablas 4, 5 y 6 (una por modalidad) se muestra el porcentaje de horas teóricas y horas prácticas sobre el total de horas del BC y del BE y se muestra la diferencia con el porcentaje correspondiente a las EM.

La gran mayoría de las CCAA, en el BC y el BE en todos los niveles formativos, han dado proporcionalmente más importancia a las horas prácticas, en relación al total de horas de cada bloque y en comparación con la proporción establecida en las EM. Únicamente es excepción Cataluña en el BC de las formaciones de TDN2 y TDS de las tres modalidades.

Analizando el BC, las CCAA que más diferencia de porcentaje presentan a favor de las horas prácticas respecto al correspondiente de las EM son, para las tres modalidades, Andalucía (TDN1), Comunidad de Madrid (TDN2) y Euskadi (TDS). Por lo que se refiere al BE, tenemos las mayores diferencias en Andalucía en todos los niveles de todas las modalidades, excepto en el TDS de esquí de fondo, en el cual Asturias presenta las mayores diferencias (ver los datos resaltados en negrita en Tabla 4, Tabla 5 y Tabla 6).

La mayor diferencia entre el porcentaje dedicado a horas prácticas por una CCAA y el porcentaje dispuesto en las EM la encontramos en el BE del TDS de snowboard por parte de Andalucía (diferencia entre porcentajes de 33,0; ver Tabla 6). La mayor diferencia si se analiza el BC la encontramos en el TDN2 de las tres modalidades por parte de la Comunidad de Madrid (diferencia entre porcentajes de 27,5; ver Tabla 4, Tabla 5 y Tabla 6).

\section{Orientaciones metodológicas}

Los centros deberán impartir las enseñanzas con arreglo a las «orientaciones metodológicas que se establezcan por el Ministerio de Educación y Cultura o por los órganos competentes de las CCAA» (RD319/2000: Cap. 2, Art. 12). Cataluña, Andalucía y Cantabria no dedican ningún artículo ni apartado a las orientaciones metodológicas. Por un lado, Aragón, la Comunidad de Madrid y Euskadi incorporan el mismo párrafo para describir cómo debe ser la metodología usada en las formaciones: «la metodología promoverá en el alumnado una visión global y coordinada de los procesos en los que tiene que intervenir, mediante la necesaria integración de los contenidos científicos, técnicos, prácticos, tecnológicos y organizativos de las enseñanzas» (Orden de 14 de diciembre de 2001: Art. 12; Orden 5094/2003: Art. 8; 172/ 2010: Art. 8). Por otro lado, Asturias y Castilla y León han desarrollado de manera más extensa este apartado; en un anexo específico, en el caso de Asturias (27/2007: Anexo 2.A), y en un apartado desarrollado en cada anexo en el caso de Castilla y León (64/2010: Anexo 1, Anexo 2 y Anexo 3). La importancia que los desarrollos normativos de las distintas CCAA han dado a la metodología y orientaciones metodológicas presenta notable heterogeneidad.

\section{Discusión}

Existen estudios similares que han comparado la normativa estatal y el desarrollo normativo de las CCAA por lo que se refiere a la legislación del turismo activo en España. El ordenamiento jurídico entre las diferentes CCAA «es diverso y casi tan heterogéneo como normas han sido legisladas» y «resulta difícil [...] entrever una línea convergente de carácter general en la regulación del ámbito en los diferentes territorios» (Inglés y Seguí, 2012a, pp.193-194). Los mismos autores reportan que «cada CCAA ha regulado lo que ha querido y como lo ha querido en función de sus competencias y objetivos» (2012a, p.163). Dicha información coincide con los resultados del presente estudio, los cuales evidencian notable heterogeneidad entre las normativas autonómicas.

Del mismo modo que las CCAA han regulado de manera diversa las formaciones técnicas en la especialidad de deportes de invierno, en el ámbito de las enseñanzas deportivas se constata «la necesidad de revisar diferentes cuestiones como la diversidad en la implantación por CCAA» (Madrera, et al., 2015, p.153) y, en referencia a las ADMN, en general, se reporta una «falta de claridad y consenso en la legislación» referente a las ADMN «por su juventud en cuanto a ser tratadas en procesos legales» (Inglés y Seguí, 2012b, p.94), evidenciando una vez más heterogeneidad dentro del territorio español.

En el ámbito de la Educación Física en Educación Primaria y la Educación Secundaria Obligatoria, sucede algo parecido a las EDRE a nivel de desarrollo curricular, ya que «el gobierno español genera un contexto curricular general y flexible para la Educación Física, de forma que pueda ser modificado, de manera posterior, por las CCAA, así como por las propias escuelas» (López-Gil et al., 2019, p.536). Trabajos previos han comparado el desarrollo curricular de educación física que las CCAA han realizado a partir de la normativa estatal reguladora, concluyendo una «elevada disparidad entre los documentos curriculares de las distintas CCAA» (Méndez, Fernández-Río, Méndez, y Prieto, 2015, p.17), «entre las autonomías en la manera de abordar los elementos curriculares» $\mathrm{y}$ «disparidad en las interpretaciones realizadas por las CCAA en el desarrollo y concreción curricular» (Méndez et al., 2017, p.82).

Por un lado, la atribución de competencias a las CCAA «tiene [...] la ventaja de permitir la adaptación de la legislación turística a las actividades que se practican en cada zona» (Nasarre, 2000, p.72). La «diversidad [... ] entendida desde el punto de vista de que cada CCAA ha legislado en función de sus competencias y en relación a sus necesidades y realidad» puede ser considerada positiva y ser una «clara manifestación de la España de las CCAA» (Inglés y Seguí, 2012a, p.194). En el panorama educativo formal de la Educación Física, esta “opcioìn de flexibilidad curricular» se puede entender como positiva, «ya que proporciona mayor capacidad de decisioìn a las CCAA, a los centros educativos y a los propios docentes a la hora de escoger el curriìculo necesario seguìn el contexto» (López-Gil et al., 2019, p.536).

Por otro lado, Nasarre estudió también la regulación jurí- 
dica de las empresas de TA en España aportando que las desigualdades que se producen en la legislación entre las diferentes CCAA «resultan en ocasiones difícilmente explicables, al ser diferentes los requisitos exigidos [...] a uno y otro lado de los límites autonómicos» (2000, pp.71-72) y, unos años más tarde, se continúa evidenciando una necesidad de «convergencia de normativas» (Nasarre, 2008, p.24) en el sector. Inglés y Seguí sugieren una idea similar: «tanta heterogeneidad dificulta la promoción del TA o las ADMN a nivel interterritorial» (2012a, p.194), conclusiones que coinciden con el presente estudio: existencia de heterogeneidad de la regulación entre las diferentes CCAA en contraposición con la validez estatal de la titulación. En el ámbito de la Educación Física en primaria y secundaria, sucede algo similar: la autonomía pedagoìgica que se estableció con la LOE «parece maìs un libre albedriìo que una forma de generar la posibilidad de adecuacioìn a las caracteriìsticas de la comunidad, del contexto y del alumnado» (Méndez et al., 2017, p.86) y podría suponer una «pérdida de la estabilidad curricular garantizada en las asignaturas troncales para todo el territorio nacional» (Méndez et al., 2017, p.82). Es por dichos motivos que el presente estudio hace evidente la necesidad de converger en los aspectos que se tratan a continuación.

Se ha constatado pues la falta de pleno consenso entre normativa estatal y normativas de las CCAA por lo que se refiere a unidades de competencia y competencias profesionales en las titulaciones. Estudios previos referidos al espacio profesional de la actividad física y el deporte evidencian una «necesidad de [...] profesionalización, asociada a una carencia de formación y capacitación por parte de las personas que están ejerciendo funciones de actividad física y deporte», hecho que deviene un "problema grave» que tiene por solución «la articulación de un desarrollo legislativo» (Martínez, et al., 2012, p.55).

El presente estudio considera las asignaturas cursadas, la distribución horaria y el repartimiento de horas teóricas y prácticas como una principal línea a converger en las regulaciones de las titulaciones de TD y TDS en los deportes de invierno. Esta idea se ve soportada por Sáez y Giménez, quienes concluyeron la necesidad de la existencia de «una institución que coordine, legisle, oriente y desarrolle programas formativos adecuados para todos los niveles» para asegurar que los contenidos tengan la misma validez y la misma estructura básica «independientemente de la institución que lo lleve a cabo» (2005, p.17) por lo que se refiere al conjunto de formaciones relacionadas con las ADMN. Centrando la atención en la Educación Física escolar (Primaria y Secundaria) se reportó también, en los bloques de contenido de los desarrollos del currículo, una «concreción muy dispar entre unas y otras CCAA» (Méndez et al., 2015, p.17). Madrera et al. coincidieron en que «sólo a través de una buena concreción legislativa se conseguirá una forma definida de enseñar y evaluar cada contenido reflejado en las enseñanzas conducentes a titular como técnico deportivo» (2015, p.155).

La anteriormente descrita heterogeneidad en la regulación concuerda con las conclusiones del análisis del proyecto Vocasport, que ha estudiado la situación de la formación profesional deportiva en los distintos países de la Unión Europea. Reporta una «diversidad de los sistemas y políti- cas de educación y formación profesional en el campo del deporte» y sus observaciones «muestran una gran diversidad en las formas de las regulaciones implementadas en el campo de la formación profesional para las profesiones deportivas» (Projet Vocasport, 2004, p.120); una diversidad y heterogeneidad que supera las fronteras del estado español y se refiere al conjunto de la Unión Europea.

\section{Conclusiones}

En el año 2018, ocho son las CCAA (Aragón, Cataluña, Comunidad de Madrid, Andalucía, Principado de Asturias, País Vasco, Cantabria y Castilla y León) que han desarrollado normativa específica relativa a las titulaciones de TD o TDS en la especialidad de los deportes de invierno, 18 años después de la publicación de la normativa específica estatal. Las ocho CCAA han regulado las formaciones de TD y TDS excepto Cataluña, quién hasta el momento cuenta únicamente con la regulación de las titulaciones de TD. Los hechos más destacados que evidencian la heterogeneidad de las normativas autonómicas al respecto son los siguientes.

La mayoría de las CCAA citan textualmente las unidades de competencia y las capacidades profesionales dictadas en el RD319/2000 excepto Cataluña (incorpora cambios relevantes a nivel de contenido) y Euskadi (incorpora cambios relevantes únicamente en el TDN1 de esquí alpino). Este hecho podría devenir generador de problemas a un lado y otro de un límite autonómico. Por lo que se refiere al ejercicio de la actividad profesional, no existe pleno consenso entre normativa estatal y normativas de las CCAA; Cataluña y Andalucía clarifican, añaden y concretan aspectos en sus normativas específicas comparado con lo expuesto en el RD319/ 2000. Por otro lado, sí existe homogeneidad entre las CCAA y el Real Decreto en lo que se refiere a la tipología de entidades y empresas dónde ejercer la profesión.

En referencia a las asignaturas que constituyen los planes de estudio, existen algunas que no aparecen en el RD319/ 2000 y únicamente se cursan en una CCAA (por ejemplo, «Euroseguridad» en el TDN1 y TDN2 de Aragón y «Reglamento y cronometraje» en el TDN2 de Cataluña). Existen asignaturas que en una CCAA se cursan en un nivel formativo distinto a otras CCAA. Esta heterogeneidad puede generar que se formen técnicos/as en diferentes CCAA con planes de estudio muy distintos pero que podrán ejercer con una misma titulación de validez estatal.

A nivel de distribución horaria y carga lectiva, se puede observar heterogeneidad en la distribución horaria que han establecido las diferentes CCAA para una misma formación. Es necesario destacar, también, que únicamente Euskadi ha igualado la carga lectiva total de las tres modalidades, mientras que en las otras CCAA ésta varía en función de cada modalidad (siguiendo la línea marcada en el RD319/2000). Este hecho quiere decir que, para obtener la misma titulación, el alumnado dedica a los estudios más o menos horas dependiendo de la CCAA. Por lo que se refiere a la distribución de horas prácticas y teóricas, la gran mayoría de las CCAA (en todos los niveles formativos, y tanto en el BC como en el BE) ha dado más importancia a las horas prácticas que a las teóricas en comparación con la proporción establecida en las EM del RD319/2000. Andalucía es, por lo general, 
la que más diferencia de porcentaje presenta a favor de las horas prácticas respecto al correspondiente establecido en las EM. La importancia que se da a las orientaciones metodológicas para la impartición de las formaciones en los desarrollos normativos es heterogénea entre las diversas CCAA.

De este modo, por lo general, se ha podido constatar la destacada heterogeneidad en la regulación de las formaciones de las diferentes CCAA.

El presente análisis ha evidenciado, entonces, la urgente necesidad de homogeneización de los desarrollos normativos de la formación de TD y TDS en la especialidad de deportes de invierno. Se considera que las principales líneas a converger, según lo expuesto, son las unidades de competencia y capacidades profesionales, el ejercicio de la actividad profesional, las asignaturas cursadas, la distribución horaria, la distribución de horas prácticas y teóricas y las orientaciones metodológicas para la impartición de las formaciones, con el objetivo de asegurar una convergencia para el ejercicio profesional de estas modalidades en todo el territorio estatal.

El presente estudio basa su análisis en el contenido de los textos que conforman el ordenamiento jurídico Estatal y de las CCAAy, por lo tanto, no se ha incluido en el análisis la valoración que hacen las empresas, los equipos de dirección de escuelas de formación, las federaciones, el alumnado y el profesorado al respecto. Sin duda, el contraste de los textos normativos con su visión, mediante datos cualitativos en un futuro estudio, arrojarán luz a la comprensión global de la temática.

Por otro lado, el análisis de la especialidad de deportes de invierno ha servido para evidenciar claramente la heterogeneidad en la regulación de estas modalidades. Aun así, se considera de especial interés ampliar el estudio al análisis de la normativa específica de otras especialidades deportivas (por ejemplo, deportes de montaña y escalada) e incluso de todas las especialidades de formaciones técnicas en ADMN.

\section{Agradecimientos}

Con el apoyo del Institut Nacional d’Educació Física de Catalunya (INEFC) de la Generalitat de Catalunya.

\section{Referencias}

Avellanas, M.L. (1995). Los accidentes de montaña en España: Análisis de la situación actual, sobre un estudio epidemiológico de los últimos 25 años (1969-1993). Tesis doctoral, Universidad de Zaragoza, Zaragoza

Bonnet, M., Wehbe-Herrera, C., y Lobo, Á. (2018). Analysis of the Regulation of Active Tourism in Spain. Revista de Estudios Empresariales. Segunda Época, 2(2), 28-56. /doi.org/10.17561/ ree.v2018n2.2

Camps, A., Carretero, J.L., y Perich, M.J. (1995). Aspectos normativos que inciden en las actividades físico-deportivas en la naturaleza. Apunts, educación Física y Deportes, 41, 44-52

Departament d'Ensenyament, Generalitat de Catalunya (2018). Documents per a l'organització i la gestió dels centres. Cicles esportius LOE i LOGSE. Tomado el 3 de marzo de 2019 de http:// educacio.gencat.cat/documents/IPCNormativa/DOIGC/ CUR_Esports.pdf

González, F. (2011). El sistema nacional de cualificaciones y formación profesional. Madrid: Editorial Vértice

Inglés, E. y Seguí, J. (2012a). Estudio comparativo del ordenamiento jurídico del turismo activo y las actividades deportivas en el medio natural en la España de las comunidades autónomas. Acciones $e$
Investigaciones Sociales, 31, 159-200

Inglés, E. y Seguí, J. (2012b). La responsabilidad civil en las actividades en el medio natural en la comunidad autónoma de Cataluña. Medidas de prevención. Apunts. Educación física y deportes, 3 (109), 89-97. doi.org/10.5672/apunts.2014-0983.es.(2012/3).109.09

Instituto Nacional de las Cualificaciones y Consejo Superior de Deportes (2007). La Familia Profesional de las Actividades Físicas y Deportivas. Colección Informes. Tomado el 5 de marzo de 2019 de https://sede.educacion.gob.es/publiventa/d/14500/19/0

López, S., Maneiro, R., Moral, J. E., Amatria, M., Diez, P., Barcala, R., \& Abelairas, C. (2019). Los riesgos en la práctica de actividades en la naturaleza. La accidentabilidad en la Educación Física, en las prácticas deportivas y medidas preventivas. Retos: Nuevas Tendencias En Educación Física, Deporte y Recreación(36), 618-624

López-Gil, J. F., Devantel, H., \& Renato, F. (2019). Análisis cualitativo de la educación física escolar en España y Brasil: realidades, similitudes, diferencias y propuestas de mejora. Retos: Nuevas Tendencias En Educación Física, Deporte y Recreación(36), 535-542

Madrera, E.; García, J.; Lambea, E.; Martínez, J.A.; García, J.R.; Salesa, R.; ... y Romanos, L. (2014). Las enseñanzas deportivas en el contexto actual de algunos centros públicos de formación de técnicos: hacia un modelo común de organización. Revista REEFD, 405, 77-90

Madrera, E.; Garrido, A., y González, L.E. (2015). La formación de profesionales de las actividades físico-deportivas en la enseñanza nouniversitaria. Retos: Nuevas tendencias en Educación Física, Deporte y Recreación(27) 152-158

Martínez, G., Campos, A., González, M.D., y Viaño, J. (2012). Las titulaciones de actividad física y deporte de las personas que trabajan en instalaciones turísticas, residenciales y naturales en España. Revista Digital de Educación Física, 17, 49-58

Mediavilla, L. y Villota, S. (2012). Percepción de los riesgos y las causas de los accidentes en el medio natural por parte de deportistas, gestores, grupos de rescate y visistantes. Acciones e investigaciones sociales, 31, 39-61

Méndez, D., Fernández-Río, J., Méndez, A., y Prieto, J. A. (2015). Análisis de los currículos autonómicos LOMCE de Educación Física en Educación Primaria. Retos: Nuevas Tendencias en Educación Física, Deporte y Recreación(28), 15-20

Méndez, D., Pérez, A., Méndez, A., Fernández, F. J., y Prieto, J. A. (2017). Análisis del desarrollo curricular de la Educación Física en la Enseñanza Secundaria Obligatoria. Comparación de los currículos autonómicos. Retos: Nuevas tendencias en Educación Física, Deporte y Recreación(31), 82-87

Nasarre, J.M. (2000). La regulación jurídica de las empresas de turismo activo. Acciones e Investigaciones Sociales, 10, 67-82

Nasarre, J.M. (2008). La regulación jurídica de las empresas de turismo activo. Zaragoza: Prames

Nerín, M.A. y Morandeira, J.R. (2005). Estado actual de la prevención de los accidentes de montaña en Aragón. Cultura, ciencia y deporte: revista de ciencias de la actividad física y del deporte de la Universidad Católica de San Antonio, (2), 75-86

Parlamento Europeo y Consejo de la UE (2008). EQF-MEC Marco Europeo de cualificaciones para el aprendizaje permanente, 2008/ C 111/01. Recuperado de https://ec.europa.eu/ploteus/sites/eac-eqf/ files/journal_es.pdf

Powell, C. (2007). The perception of risk and risk taking behavior: Implications for incident prevention strategies. Wilderness \& environmental medicine, 18(1), 10-15

Projet Vocasport (2004). Améliorer l'emploi dans le domaine du sport en Europe par la formation professionnelle. Proyecto sostenido por la Comisión Europea. DG de Educación y Cultura. Tomado el 1 de febrero de 2019 de http://francepleinair.fr/wp-content/uploads/2015/ 10/VOCASPORT_Final_Report_fr1.pdf

Sáez, J. y Giménez, F.J. (2005). Análisis de la oferta formativa en actividades en el medio natural. Lecturas: Educación física y deportes, 83. Tomado el 2 de febrero de 2019 de https://www.efdeportes.com/ efd83/mnatural.htm

Sánchez, A. (2016). Siniestralidad en los deportes de montaña. Realidades y soluciones. Tesis doctoral, Universidad de Zaragoza, Zaragoza

Vela, P. (2009). Epidemiología de los accidentes de montaña en el Pirineo Aragonés que no precisan rescate durante la temporada estival. Fundación Mapfre, Instituto de Prevención, Salud y Medio Ambiente

Wild, F.J. (2008). Epidemiology of mountain search and rescue operations in Banff, Yoho, and Kootenay National Parks, 2003-06. Wilderness \& environmental medicine, 19(4), 245-251. 